OPEN ACCESS

Edited by:

Devin William McBride, University of Texas Health Science Center at Houston, United States

Reviewed by: Pio Conti,

University of Studies G. d'Annunzio

Chieti and Pescara, Italy Vadim V. Sumbayev, University of Kent, United Kingdom

${ }^{*}$ Correspondence: Gao Chen d-chengao@zju.edu.cn

${ }^{\dagger}$ These authors have contributed equally to this work and share first authorship

Specialty section: This article was submitted to Multiple Sclerosis and Neuroimmunology, a section of the journal Frontiers in Immunology

Received: 27 September 2021 Accepted: 26 October 2021 Published: 16 November 2021

Citation:

Wu X, Zeng $H$, Cai $L$ and Chen $G$ (2021) Role of the Extracellular Traps in Central Nervous System.

Front. Immunol. 12:783882. doi: 10.3389/fimmu.2021.783882

\section{Role of the Extracellular Traps in Central Nervous System}

\author{
Xinyan $\mathrm{Wu}^{\dagger}$, Hanhai Zeng ${ }^{\dagger}$, Lingxin Cai and Gao Chen * \\ Department of Neurological Surgery The Second Affiliated Hospital, Zhejiang University School of Medicine, Hangzhou, China
}

It has been reported that several immune cells can release chromatin and granular proteins into extracellular space in response to the stimulation, forming extracellular traps (ETs). The cells involved in the extracellular trap formation are recognized including neutropils, macrophages, basophils, eosinophils, and mast cells. With the development of research related to central nervous system, the role of ETs has been valued in neuroinflammation, blood-brain barrier, and other fields. Meanwhile, it has been found that microglial cells as the resident immune cells of the central nervous system can also release ETs, updating the original understanding. This review aims to clarify the role of the ETs in the central nervous system, especially in neuroinflammation and bloodbrain barrier.

Keywords: extracellular traps, neuroinflammation, blood-brain barrier (BBB), stroke, neurodegenaration, CNS

\section{INTRODUCTION}

Extracellular traps (ETs) were first found in neutrophils and regarded as a host defense in response to bactericidal proteins and peptides (1). ETs were also considered to play a role in the inappropriate clearance of dead and dying cells in systemic lupus erythematosus (SLE) (2). They existed in a polynucleosome form, where histones associated with DNA tightly (3). Neutrophil extracellular traps (NETs) are large, extracellular, web-like structures of cytoplasmic and granular proteins clustered on a scaffold of decoagulated chromatin (1). Nuleus is the majority origination of the NET DNA, but mitochondrial DNA is also involved in the composition of NETs (1). According to the current research, the role of NETs is not limited to preventing microbial invasion but also participation in immune-related diseases $(1,4-8)$. With the development of research, more cells have been found to be involved in the formation of ETs (Figure 1). The other leukocytes including mast cells, eosinophils, and basophils also have been found to produce extracellular traps (9-13). The formation of ETs seems to play an alternative role in defense when the phagocytic capacity of cells is overtaxed (8). The characteristic of ETs is the DNA release related to histones and granule proteins, forming an extracellular web-like structure (14). This structure can capture and kill some microorganisms by acting as an immune defense (15). On the one hand, ETs can protect the body in response to the invasion of pathogenic microorganism. On the other hand, the excessive release of ETs can cause adverse effects in some diseases such as autoimmune diseases, cancers, and so on (1621). Thus, the balance between the protective ET formation and the efficient elimination of excessive ETs still needs to be considered. Recently, the significant role of ETs in the central nervous system has come to be recognized in various related diseases. In this review, we described the development trend of ETs and the crosstalk between ETs and peripheral or central immune system. 


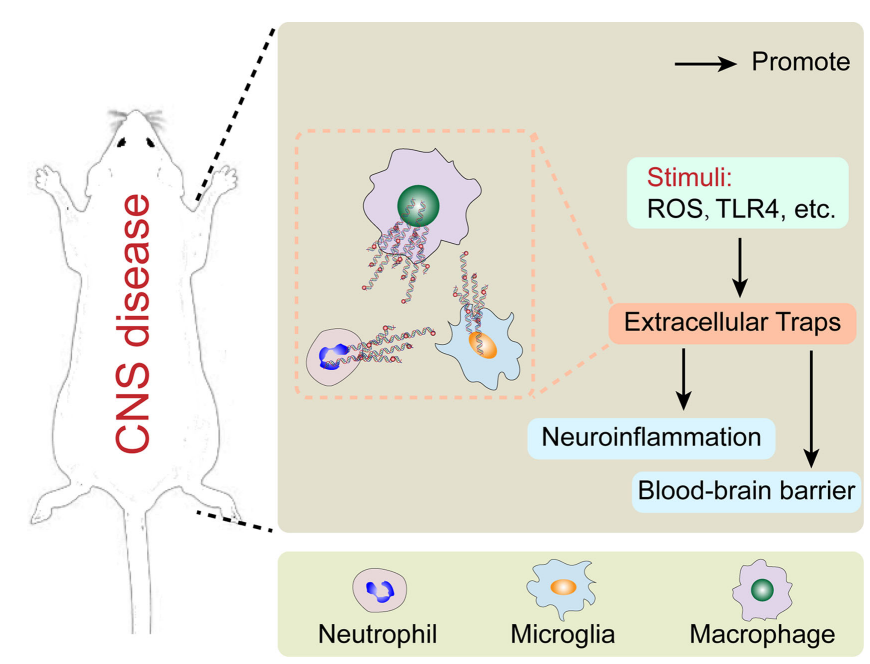

FIGURE 1 | The major cells forming extracellular traps in central nervous system disease and their potential mechanism.

\section{NETs}

Neutrophils have specialized in the formation of NETs, which are the most studied type of extracellular traps. As mentioned above, NETs can fight against microbes through immobilizing function and their antimicrobial compound equipment, which means the physiological functions of NETs include immune defense and autoimmunity. Moreover, the evidence for the inflammation-regulation action of NETs has been accumulating $(22,23)$. It has shown that the higher density of NET effect is a double-edged sword: on one hand, aggregated NETs can isolate the blocks of materials with immunostimulatory activity, leading to the limitation of immune reactivity and inflammation to sterile agents (22, $24,25)$. On the other hand, tissue damaging is also one of the properties of NETs, suggesting that the higher density of NETs can result in an enlarged injury to tissue (25-27). The existing studies have highly assumed that neutrophil aggregation and NET formation might be interdependent, but the specific relationship and mechanisms between them remain unclear. As crucial cells of innate immunity, neutrophils are seldom found in the central nervous system (CNS) under normal conditions because of the presence of the blood-brain barrier (BBB). However, neutrophils can be activated in response to CNS diseases or exogenous stimulus and then injure the BBB. Meanwhile, the infiltration of neutrophils and the release of NETs increase. This phenomenon has been reported to exist in various neurological diseases, such as stroke, traumatic brain injury (TBI), neurodegenerative diseases, autoimmune diseases, and tumors. In related diseases, NETs always play a role of aggravating diseases through its properties and damaging the integrity of BBB (Figure 2). The following are classified according to different types of diseases and will show how the neuroinflammation and $\mathrm{BBB}$ damage caused by NETs influence the concrete neurological diseases.

\section{ACUTE ISCHEMIC STROKE}

Acute ischemic stroke is a hypoxic-ischemic disorder associated with a sterile inflammatory reaction (28), promoting immune cell migration and infiltration to the brain parenchyma $(29,30)$. Animal studies showed that neutrophils infiltrated the ischemic areas of the brain within a few hours after the onset of experimental ischemia, and Perez-Puig et al. described the presence of citrullinated histone 3 , a hallmark of NET formation, in the ischemic brain after 24-h ischemia (31). Peña-Martínez et al. indicated that the neutrophil activation by platelet Toll-like receptor 4 (TLR4) could result in NETosis and NETs could act as assembly platforms for atherothrombosis by binding platelet-derived microparticles (PMPs) and clotting factors (32-34). Recent research considered that NETs promoted vaso-occlusion, and this process was initiated through von Willebrand factor ( $\mathrm{vWF})(35,36)$. Meanwhile, it has been reported that the outer shell of thrombus samples from clinical acute ischemic stroke (AIS) patients acting as a protective barrier against thrombolysis are composed of fibrin, RBC, vWF, leukocytes, and nucleated cells (37-41), which suggest the potential value of therapy targeting NETs in thrombolysis. Additionally, DNAse 1 has been shown to target NETs and extracellular DNA in thrombi retrieved from patients with AIS and then accelerates ex vivo lysis of cerebral thrombi $(36,42,43)$.

The existing research indicated that NETs play a role in neurological dysfunction after ischemia probably through the BBB destruction $(31,44)$. It has been reported that neovascularization and perfusion of the vascular structure in the peri-ischemic brain have important roles in stroke recovery $(45,46)$, while NETs can release many cytotoxic proteases such as histone, elastase, and MPO, which directly induce endothelial cell damage to increase vascular permeability and then break BBB (44). Kim et al. demonstrated that high-mobility group box-1 (HMGB1), a prototypic danger-associated molecular pattern (DAMP), is involved in NET-mediated neuronal 


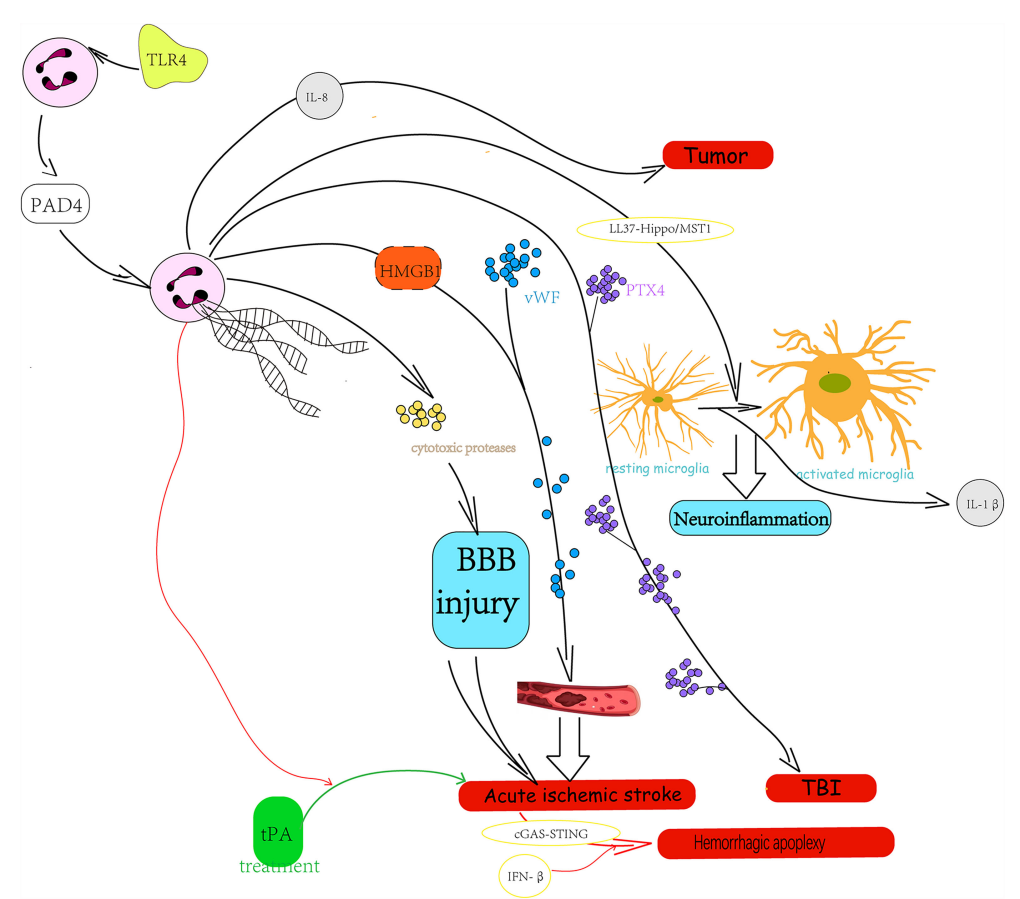

FIGURE 2 | The role and possible mechanism of neutrophil extracellular traps in central nervous system disease.

damage in the ischemic brain, where disulfide HMGB1 can induce NETosis via CXCR4 and TLR4 (47, 48). The experiments by Kim et al. are also the first to describe the temporal and spatial progressions of NETosis after middle cerebral artery occlusion (MCAO) using an intraluminal model and report that the main route of neutrophil infiltration from peripheral blood vessels after brain ischemia follows the route: leptomeningeal vessel $\rightarrow$ Virchow-Robin space $\rightarrow$ perivascular space $\rightarrow$ brain parenchyma (47).

Although the thrombolysis with tissue plasminogen activator (tPA) is the only approved pharmacological therapy for acute ischemic stroke, it presents a major clinical problem as an increased risk of intracerebral hemorrhage (49-51). Ranran et al. also revealed that $\mathrm{PPA}$ treatment in mice with thrombotic stroke increased NET formation and that activation of the cGASSTING pathway and production of IFN- $\beta$ participated in NETmediated effects on tPA-associated cerebrovascular complications in stroke (52). These NETs were related to the increased risk of intracerebral hemorrhage, all of which could be attenuated by clearing NETs with DNase I or inhibiting NET production by PAD4 deficiency (52). The series of research results mean the potential therapeutic value of targeting NETs in both thrombolysis and the adjuvant therapy of tPA.

Meanwhile, the present studies have shown that neutrophil influx is more prominent after permanent than after transient MCAO and the frequency and intensity of NETosis are significantly greater after permanent MCAO, which suggests a relationship between NETosis and disease severity $(31,47,53)$. Moreover, it was observed that NET marker levels were associated with stroke severity at onset and discharge from hospital as evaluated using NIHSS and mRs scores in the plasma of acute ischemic stroke patients, and that increasing levels of $\mathrm{CitH} 3$ at onset were associated with all-cause mortality at 1-year follow-up visits (54). Therefore, NETs may be a useful prognostic maker in acute ischemic stroke in the future according to the current research results.

\section{HEMORRHAGIC APOPLEXY}

The proportion of nontraumatic intracerebral hemorrhage (ICH) in acute strokes is $\sim 10 \%-15 \%$, and nontraumatic ICH has a much higher risk of mortality than ischemic strokes or subarachnoid hemorrhage $(55,56)$. A recent research reported the NET infiltration in the brain of patients who died from spontaneous intracerebral hemorrhage $(\mathrm{sICH})$, suggesting that NETs might interact with early hemostasis within the hematoma core and with the surrounding neuroinflammatory response (57). Orbán-Kálmándi et al. pointed out that a modified clot lysis assay, incorporating the effect of NETs, could suggest unfavorable outcomes in spontaneous, nontraumatic ICH based on a prospective observational study data (58). Also, in subarachnoid hemorrhage (SAH), the current studies revealed that NETs can induce the alteration of microglia into a proinflammation subtype in order to promote neuroinflammation and cause adverse consequences, suggesting that NETs may be a potential target for the treatment SAH in its early phase $(59,60)$. According to available research, we can make a bold assumption 
that NETs may be a potentially effective therapeutic target to hemorrhagic apoplexy.

\section{TRAUMATIC BRAIN INJURY}

TBI is a major public health issue, which may contribute to elevated intracranial pressure (ICP) and lead to neurological deterioration. A research identified NETs as the distinct mediators of cerebral edema which caused elevated ICP and neurological deterioration (61). The researchers revealed that the formation of NETs was induced by the activation of TLR4 via a PAD4-dependent mechanism and then resulted in neurological deficits (61). In 2021, researchers have demonstrated the presence of NETs in paraventricular nucleus (PVN) after TBI and NETs activated microglia dependent on the LL37-Hippo/ MST1 pathway to facilitate the IL-1 $\beta$ release, which may lead to the occurrence of sympathetic excitation as a result (62). Oggioni et al. reported the presence of PTX3 in the mouse brain parenchyma, next to astrocytes, neurons, microglia, and endothelial cells, in the subacute phase of TBI for the first time (63). In view of the complex functions of PTX3 in different cells (64-67), this research considered that PTX3 may alleviate subacute pathological sequelae after TBI (63). Meanwhile, PTX3 is complexed with the NET components, which suggest that NETs may participate in the subacute phase of TBI through PTX3.

\section{NEURODEGENERATIVE DISEASES}

Alzheimer's disease $(\mathrm{AD})$ is one of the neurodegenerative disorders characterized by the progressive deterioration of cognitive functions. Its neuropathological features include amyloid- $\beta$ (A $\beta$ ) accumulation, the formation of neurofibrillary tangles, and the loss of neurons and synapses (68). Neuroinflammation is a well-established feature of AD pathogenesis, and recent studies have identified several inflammation pathway genes associated with the risk of $\mathrm{AD}$ $(69,70) . \mathrm{AD}$ is also characterized by the loss of $\mathrm{BBB}$ integrity, which disrupts the clearance of $A \beta$ and thus promotes $A \beta$ accumulation in the brain, leading to neuronal injury and cognitive decline (71). Recent research have indicated that both the intravascular NETs and intraparenchymal NETs have an impact on $\mathrm{AD}$ (72). Zenaro et al. reported the existence of neutrophil-microglia crosstalk and intravascular and intraparenchymal NETs in AD in 2015 (73). This existence suggested that NETs can possibly damage the $\mathrm{BBB}$ and neurons in $\mathrm{AD}(73,74)$. Additionally, the research supported the neutrophil-dependent brain damage in $\mathrm{AD}$, showing that the migration of neutrophils produced IL-17 which has toxic effects on neurons directly and may recruit more neutrophils (73). In an $\mathrm{AD}$ mouse model, it has been found that the block of LFA-1 integrin can reduce the neutrophil adhesion in the brain microvasculature, alleviating the cognitive deficits (73). Interestingly, previous studies reported that the brain vasculature in $\mathrm{AD}$ humans produced more cytokines such as TNF- $\alpha$, IL- $1 \beta$, IL- 8 , and thrombin which triggered the intravascular NETs compared with the age-matched controls (75-80). A recent research has reported the presence of three times excessive level of NET formation in the peripheral blood of mild cognitive impairment (MCI) patients who are the precursor of $\mathrm{AD}$ and a positive correlation between the excessive level of NET formation and the content of $A \beta(81)$. In vitro studies based on brain endothelial cells revealed increasing expression of cytokine genes which induce NETosis in response to the exposure to $A \beta$ peptides (82). Moreover, $A \beta$ peptides can further promote intravascular NETosis by inducing the generation of ROS and secreting more proinflammatory cytokines (72). Neutrophils invaded the brain parenchyma at the early stage of $\mathrm{AD}$ in mice model and produced NETs, leading to memory deficit (73). Similarly, the release of intraparenchymal NETs can also be influenced by related cytokines as intravascular NETs in the case of A $\beta$ peptide exposure $(73,83)$. Meanwhile, a study pointed out that the intraparenchymal release of NETs can also respond to other fibrillary form of amyloids (84). The zurophilic granules of neutrophils release MMPs and serine proteases which can cause tissue injury and exacerbate the inflammatory response during the generation of NETs. MMPs activated at the stimulation of neutrophils are involved in the proteolysis of the extracellular matrix in order to cause damage to the brain parenchyma (85). The serine proteases such as NE can also degrade tissues not only by cleaving extracellular matrix proteins but also by inactivating the endogenous tissue inhibitors of MMPs (TIMPs) which can also be inhibited by MPO localized within NETs $(86,87)$. The existing research suggests that NETs seem to be a new therapeutic target to ease the $\mathrm{AD}$ progression, and more research is still needed to investigate the mechanism of NETs in AD (88).

In addition to $\mathrm{AD}$, Parkinson's disease is also a kind of neurodegenerative disorder resulted from the accumulation of amyloid fibrils formed by specific misfolded proteins (89). And in amyloid diseases, researchers observed that amyloid fibrils induced NETs release dependent on the NADPH oxidase system to a large extent (84). Although there are few studies focusing on the other neurodegenerative disorders, the study based on related pathomechanism has been proceeded, suggesting the potential therapeutic value of NETs in neurodegenerative disorders.

\section{AUTOIMMUNE DISEASES}

NETs were considered to contribute to the autoimmune diseases as early as the phenomenon of NET release was first reported (1). Current researches have shown that NETs are closely related to a of lot of autoimmune diseases including those that may be affecting the central and peripheral nervous systems (90-92). Multiple sclerosis (MS) is a chronic inflammatory, demyelinating disease, and the mechanism of MS includes both a complex genetic trait and environmental factors (93). As mentioned in a recent review, NETs may have a cytotoxic effect on the BBB and facilitate the 
damage of adjacent neurons and other cells of the CNS in MS (94). Meanwhile, the NET-associated decrease of proteins can ease the MS progression and increase the BBB integrity $(94,95)$. Interestingly, the NETs in the serum of MS patients are elevated compared with the controls, and there is a partial sex difference in the extent of elevation of NETs $(92,95,96)$. In mice model, Allen revealed that murine neutrophil metastases through activated cerebrovascular endothelial cells could induce a proinflammatory and neurotoxic phenotype and lead to the release of NET as a result (74). Although researchers have indicated the NET-related potentiation of proteases to modify the immune complexes, the real mechanism in human MS is still unclear (97).

In addition to MS, NETs are also involved in other neuropsychiatric symptom. According to a recent review, it concludes a hypothesis for the cognitive dysfunction in systemic lupus erythematosus (SLE): MMP-9 released by prestimulated LDGs can degrade the basal lamina and damage the integrity of $\mathrm{BBB}$. And then the anti-NR2A/B antibodies further activate the $\mathrm{BBB}$, increasing the expression of endothelial cell adhesion molecules. Afterwards, the neutrophils recruit, roll, adhere and transmigrate, leading to further NETosis. Finally, the NETs release leads to neurotoxity through inducing neuron death, causing the neuropsychiatric manifestations of SLE as a result (98). In microscopic polyangiitis (MPA), it is considered that NETs may be involved in the pathogenesis of neuropathy and suggests the therapeutic strategies targeting NETs based on the nerve biopsy samples from MPA patients (99).

\section{MALIGNANT BRAIN TUMORS}

Malignant brain tumors can be both primary tumors originated in the brain such as gliomas and exogenous tumors that metastasize into the brain such as nonsmall-cell lung carcinoma (NSCLC) (100). The prognosis for both primary and metastatic brain malignancies is poor, mainly due to the limitations of standard treatments, thus researchers turn to the tumor microenvironment (101). Based on the previous studies, NETs seemed to facilitate the cytotoxic effect and inhibit the spread of cancer cells as a result of inducing epithelial and endothelia cell death $(26,102)$. On the contrary, neutrophils have been proved to facilitate the metastasis of tumor in experiments and animal studies in different cancers (103-107). In 2013, Cools-Lartigue et al. pointed out that the NETs can promote tumor metastasis through isolating circulating tumor cells (108). Moreover, the recent research reported that NETs formed during LPS- or tobacco smoke-induced lung inflammation can awaken dormant cancer cells and cause metastasis in mice dependent on FAK/ERK/MLCK/YAP pathway (109-111). In glioma cells, it has been considered that NETs could induce the expression of IL-8 which is correlated with tumor burden and prognosis through a HMGB1- and RAGE/ERK/NF- $\kappa B$ axis-dependent manner (112). Furthermore, the IL- 8 produced by glioma can cause in turn the formation of NETs (112). T-cell immunoglobulin and mucin domain-3 (TIM-3) also has been proven to interact with HMBG1 in TADCs and then preventing the nucleic acids from localizing into the endosomal vesicles, thus playing a role in blowing the antitumor effect of tumor-associated dendritic cells (TADCs) (113115). Additionally, the administration of anti-Tim-3 mAb during chemotherapy has been demonstrated to lead to tumor regression (113). Interestingly, a current research has reported that TIM-3 can suppress the uptake of extracellular DNA in intratumoral dendritic cells in order to limit the activation of cGAS-STING pathway, which may influence the production of NETs (116). While these discoveries unstated the type of tumor and the specific role of NETs in the referred mechanism, they still suggest the future research interests of NETs in malignant brain tumors relating to HMBG1-TIM-3. Toll-like receptor 2 (TLR2), one of the HMBG1 receptors, has been considered to involve in the production of NETs, and HMGB1-mediated TLR2 signaling plays a critical role in eliciting glioblastoma regression, suggesting the prospect of NETs in malignant brain tumors (117-119). Although the current research has demonstrated the role of neutrophils in glioma (107), further studies are still needed to clarify the protumor and antitumor functions of NETs in glioma.

\section{METs}

Macrophages comprise a diverse group of cells and demonstrate remarkably various functions. Macrophage functions ranged from supporting development, maintaining homeostasis, keeping immune surveillance, and regulating tissue remodeling and repair (120). The formation of METs was initially found at the stimulation of Mycobacterium tuberculosis in 2013 (121). Although the following research focused on the formation of METs in vitro and in vivo, the mechanism of the formation of METs is still unknown. It has been reported that microorganisms such as Toxoplasma gondii, Candida albicans, Staphylococcus aureus, Haemophilus influenza, Klebsiella pneumoniae, and Escherichia coli can stimulate the formation of METs (122), but METs play opposite roles in different infections caused by diverse microorganisms (123125). Pertiwi et al. reported that METs were significantly more numerous in the late stages of thrombus formation and were mostly located around and inside the lipid core, suggesting that METs involved in the thrombus formation. Although the specific role of METs in thrombus formation is unclear, this finding supports the involution of METs in noninfectious diseases (126). While the studies on the role of METs in CNS are few, the research focusing on the crosstalk between monocyte-macrophage system and CNS is not in the minority, including its effect on glial cells. Therefore, the research based on the role of METs in CNS will be more in the foreseeable future, which is supposed to become a new target.

\section{MiETs}

Microglia are a resident mononuclear phagocyte population in the CNS and are gatekeepers of CNS immunology, involved in the CNS maintenance $(127,128)$. A previous study has reported that microglia can release extracellular traps (MiETs) in response to the Listeria infection in vivo and in vitro (129). Then, a following research found that MiETs could be induced by dopamine in vitro 
and interestingly the formation of MiETs did not lead to immediate cell death (130). Although the mechanism of the formation of MiETs is unclear, the existing results suggest that the role of MiETs in CNS steady state including infection, neuroinflammation, and glioma are intriguing areas for future investigations. As one of the most important immune cells in CNS, the research of microglia has been continued endlessly. The interaction between microglia and astrocytes has been well verified in CNS diseases such as vascular, tumor, and trauma. As a result, as one of the rich mineral cells, it is of great value to continue to be explored to reveal its potential pathogenesis.

\section{CONCLUSION}

The increasing evidence shows that the presence of ETs in CNS plays different roles. In this review, we described the roles of ETs in different diseases, especially focusing on the integrity of BBB and neuroinflammation. According to a lot of existing research focusing on NETs, we have demonstrated that the excessive release of NETs involves in the breakdown of BBB integrity and facilitating the neuroinflammation through releasing metalloproteinases, proteases, cytokines, extracellular histones,

\section{REFERENCES}

1. Brinkmann V, Reichard U, Goosmann C, Fauler B, Uhlemann Y, Weiss DS, et al. Neutrophil Extracellular Traps Kill Bacteria. Science (2004) 303 (5663):1532-5. doi: 10.1126/science.1092385

2. Pisetsky DS, Jiang N. The Generation of Extracellular DNA in SLE: The Role of Death and Sex. Scand J Immunol (2006) 64(3):200-4. doi: 10.1111/j.13653083.2006.01822.x

3. Pisetsky DS, Fairhurst AM. The Origin of Extracellular DNA During the Clearance of Dead and Dying Cells. Autoimmunity (2007) 40(4):281-4. doi: 10.1080/08916930701358826

4. Urban CF, Reichard U, Brinkmann V, Zychlinsky A. Neutrophil Extracellular Traps Capture and Kill Candida Albicans Yeast and Hyphal Forms. Cell Microbiol (2006) 8(4):668-76. doi: 10.1111/j.1462-5822.2005. 00659.x

5. Saitoh T, Komano J, Saitoh Y, Misawa T, Takahama M, Kozaki T, et al. Neutrophil Extracellular Traps Mediate a Host Defense Response to Human Immunodeficiency Virus-1. Cell Host Microbe (2012) 12(1):109-16. doi: 10.1016/j.chom.2012.05.015

6. Abi Abdallah DS, Lin C, Ball CJ, King MR, Duhamel GE, Denkers EY. Toxoplasma Gondii Triggers Release of Human and Mouse Neutrophil Extracellular Traps. Infect Immun (2012) 80(2):768-77. doi: 10.1128/ iai.05730-11

7. Walker MJ, Hollands A, Sanderson-Smith ML, Cole JN, Kirk JK, Henningham A, et al. DNase Sda1 Provides Selection Pressure for a Switch to Invasive Group A Streptococcal Infection. Nat Med (2007) 13 (8):981-5. doi: 10.1038/nm1612

8. Branzk N, Lubojemska A, Hardison SE, Wang Q, Gutierrez MG, Brown GD, et al. Neutrophils Sense Microbe Size and Selectively Release Neutrophil Extracellular Traps in Response to Large Pathogens. Nat Immunol (2014) 15 (11):1017-25. doi: 10.1038/ni.2987

9. Möllerherm H, von Köckritz-Blickwede M, Branitzki-Heinemann K. Antimicrobial Activity of Mast Cells: Role and Relevance of Extracellular DNA Traps. Front Immunol (2016) 7:265. doi: 10.3389/fimmu.2016.00265

10. von Köckritz-Blickwede M, Goldmann O, Thulin P, Heinemann K, NorrbyTeglund A, Rohde M, et al. Phagocytosis-Independent Antimicrobial Activity of Mast Cells by Means of Extracellular Trap Formation. Blood (2008) 111(6):3070-80. doi: 10.1182/blood-2007-07-104018
DNA, and ROS. Furthermore, more research is demanded to be implemented to focus on the other roles of ETs in the neuroinflammation and $\mathrm{BBB}$ integrity. While the relating mechanism is still unclear, the current results suggest that ETs may become the potential therapeutic targets for CNS diseases to improve prognosis.

\section{AUTHOR CONTRIBUTIONS}

LC has organized manuscripts. All authors contributed to the article and approved the submitted version.

\section{FUNDING}

This work was supported by the National Key R\&D program of China (2018YFC1312600, 2018YFC1312603), the National Natural Science Foundation of China (Nos. 81771246, 81971099, and 81870908), TCM Science and Technology Plan of Zhejiang province (2017ZZ013), TCM Key Discipline of Zhejiang province (2017-XK-A39), and the Natural Science Foundation of Zhejiang Province (LY19H090019).

11. Morshed M, Hlushchuk R, Simon D, Walls AF, Obata-Ninomiya K, Karasuyama H, et al. NADPH Oxidase-Independent Formation of Extracellular DNA Traps by Basophils. J Immunol (2014) 192(11):531423. doi: 10.4049/jimmunol.1303418

12. Schorn C, Janko C, Latzko M, Chaurio R, Schett G, Herrmann M. Monosodium Urate Crystals Induce Extracellular DNA Traps in Neutrophils, Eosinophils, and Basophils But Not in Mononuclear Cells. Front Immunol (2012) 3:277. doi: 10.3389/fimmu.2012.00277

13. Yousefi S, Gold JA, Andina N, Lee JJ, Kelly AM, Kozlowski E, et al. CatapultLike Release of Mitochondrial DNA by Eosinophils Contributes to Antibacterial Defense. Nat Med (2008) 14(9):949-53. doi: 10.1038/nm.1855

14. Neumann A, Brogden G, von Köckritz-Blickwede M. Extracellular Traps: An Ancient Weapon of Multiple Kingdoms. Biol (Basel) (2020) 9(2):34. doi: 10.3390/biology9020034

15. de Buhr N, von Köckritz-Blickwede M. How Neutrophil Extracellular Traps Become Visible. J Immunol Res (2016) 2016:4604713. doi: 10.1155/2016/ 4604713

16. Brill A, Fuchs TA, Savchenko AS, Thomas GM, Martinod K, De Meyer SF, et al. Neutrophil Extracellular Traps Promote Deep Vein Thrombosis in Mice. J Thromb Haemost (2012) 10(1):136-44. doi: 10.1111/j.1538-7836.2011. 04544.x

17. Merza M, Hartman H, Rahman M, Hwaiz R, Zhang E, Renström E, et al. Neutrophil Extracellular Traps Induce Trypsin Activation, Inflammation, and Tissue Damage in Mice With Severe Acute Pancreatitis. Gastroenterology (2015) 149(7):1920-1931.e8. doi: 10.1053/j.gastro.2015.08.026

18. Lood C, Blanco LP, Purmalek MM, Carmona-Rivera C, De Ravin SS, Smith CK, et al. Neutrophil Extracellular Traps Enriched in Oxidized Mitochondrial DNA Are Interferogenic and Contribute to Lupus-Like Disease. Nat Med (2016) 22(2):146-53. doi: 10.1038/nm.4027

19. Cools-Lartigue J, Spicer J, Najmeh S, Ferri L. Neutrophil Extracellular Traps in Cancer Progression. Cell Mol Life Sci (2014) 71(21):4179-94. doi: 10.1007/ s00018-014-1683-3

20. Nakazawa D, Marschner JA, Platen L, Anders HJ. Extracellular Traps in Kidney Disease. Kidney Int (2018) 94(6):1087-98. doi: 10.1016/j.kint.2018. 08.035

21. Euler M, Hoffmann MH. The Double-Edged Role of Neutrophil Extracellular Traps in Inflammation. Biochem Soc Trans (2019) 47 (6):1921-30. doi: 10.1042/bst20190629 
22. Muñoz LE, Leppkes M, Fuchs TA, Hoffmann M, Herrmann M. Missing in Action-The Meaning of Cell Death in Tissue Damage and Inflammation. Immunol Rev (2017) 280(1):26-40. doi: 10.1111/imr.12569

23. Grayson PC, Schauer C, Herrmann M, Kaplan MJ. Review: Neutrophils as Invigorated Targets in Rheumatic Diseases. Arthritis Rheumatol (2016) 68 (9):2071-82. doi: 10.1002/art.39745

24. Wang J. Neutrophils in Tissue Injury and Repair. Cell Tissue Res (2018) 371 (3):531-9. doi: 10.1007/s00441-017-2785-7

25. Boeltz S, Amini P, Anders HJ, Andrade F, Bilyy R, Chatfield S, et al. To NET or Not to NET:current Opinions and State of the Science Regarding the Formation of Neutrophil Extracellular Traps. Cell Death Differ (2019) 26 (3):395-408. doi: 10.1038/s41418-018-0261-x

26. Saffarzadeh M, Juenemann C, Queisser MA, Lochnit G, Barreto G, Galuska SP, et al. Neutrophil Extracellular Traps Directly Induce Epithelial and Endothelial Cell Death: A Predominant Role of Histones. PloS One (2012) 7 (2):e32366. doi: 10.1371/journal.pone.0032366

27. Pieterse E, Rother N, Garsen M, Hofstra JM, Satchell SC, Hoffmann M, et al. Neutrophil Extracellular Traps Drive Endothelial-To-Mesenchymal Transition. Arterioscler Thromb Vasc Biol (2017) 37(7):1371-9. doi: 10.1161/ atvbaha.117.309002

28. Chen GY, Nuñez G. Sterile Inflammation: Sensing and Reacting to Damage. Nat Rev Immunol (2010) 10(12):826-37. doi: 10.1038/nri2873

29. Gelderblom M, Leypoldt F, Steinbach K, Behrens D, Choe CU, Siler DA, et al. Temporal and Spatial Dynamics of Cerebral Immune Cell Accumulation in Stroke. Stroke (2009) 40(5):1849-57. doi: 10.1161/ strokeaha.108.534503

30. Iadecola C, Anrather J. The Immunology of Stroke: From Mechanisms to Translation. Nat Med (2011) 17(7):796-808. doi: 10.1038/nm.2399

31. Perez-de-Puig I, Miró-Mur F, Ferrer-Ferrer M, Gelpi E, Pedragosa J, Justicia C, et al. Neutrophil Recruitment to the Brain in Mouse and Human Ischemic Stroke. Acta Neuropathol (2015) 129(2):239-57. doi: 10.1007/s00401-0141381-0

32. Guo Y, Zeng H, Gao C. The Role of Neutrophil Extracellular Traps in Central Nervous System Diseases and Prospects for Clinical Application. Oxid Med Cell Longev (2021) 2021:9931742. doi: 10.1155/ 2021/9931742

33. Peña-Martínez C, Durán-Laforet V, García-Culebras A, Ostos F, Hernández-Jiménez M, Bravo-Ferrer I, et al. Pharmacological Modulation of Neutrophil Extracellular Traps Reverses Thrombotic Stroke tPA (TissueType Plasminogen Activator) Resistance. Stroke (2019) 50(11):3228-37. doi: $10.1161 /$ strokeaha.119.026848

34. Zhou P, Li T, Jin J, Liu Y, Li B, Sun Q, et al. Interactions Between Neutrophil Extracellular Traps and Activated Platelets Enhance Procoagulant Activity in Acute Stroke Patients With ICA Occlusion. EBioMedicine (2020) 53:102671. doi: 10.1016/j.ebiom.2020.102671

35. Papayannopoulos V. Neutrophil Extracellular Traps in Immunity and Disease. Nat Rev Immunol (2018) 18(2):134-47. doi: 10.1038/nri.2017.105

36. Laridan E, Denorme F, Desender L, François O, Andersson T, Deckmyn H, et al. Neutrophil Extracellular Traps in Ischemic Stroke Thrombi. Ann Neurol (2017) 82(2):223-32. doi: 10.1002/ana.24993

37. Di Meglio L, Desilles JP, Ollivier V, Nomenjanahary MS, Di Meglio S, Deschildre C, et al. Acute Ischemic Stroke Thrombi Have an Outer Shell That Impairs Fibrinolysis. Neurology (2019) 93(18):e1686-98. doi: 10.1212/ wnl.0000000000008395

38. Jolugbo P, Ariëns RAS. Thrombus Composition and Efficacy of Thrombolysis and Thrombectomy in Acute Ischemic Stroke. Stroke (2021) 52(3):1131-42. doi: 10.1161/strokeaha.120.032810

39. Shi C, Yang L, Braun A, Anders HJ. Extracellular DNA-A Danger Signal Triggering Immunothrombosis. Front Immunol (2020) 11:568513. doi: $10.3389 /$ fimmu.2020.568513

40. Staessens S, François O, Desender L, Vanacker P, Dewaele T, Sciot R, et al. Detailed Histological Analysis of a Thrombectomy-Resistant Ischemic Stroke Thrombus: A Case Report. Thromb J (2021) 19(1):11. doi: 10.1186/ s12959-021-00262-1

41. Longstaff C, Varjú I, Sótonyi P, Szabó L, Krumrey M, Hoell A, et al. Mechanical Stability and Fibrinolytic Resistance of Clots Containing Fibrin, DNA, and Histones. J Biol Chem (2013) 288(10):6946-56. doi: 10.1074/jbc.M112.404301
42. Ducroux C, Di Meglio L, Loyau S, Delbosc S, Boisseau W, Deschildre C, et al. Thrombus Neutrophil Extracellular Traps Content Impair tPA-Induced Thrombolysis in Acute Ischemic Stroke. Stroke (2018) 49(3):754-7. doi: 10.1161 /strokeaha.117.019896

43. Genchi A, Semerano A, Gullotta GS, Strambo D, Schwarz G, Bergamaschi A, et al. Cerebral Thrombi of Cardioembolic Etiology Have an Increased Content of Neutrophil Extracellular Traps. J Neurol Sci (2021) 423:117355. doi: 10.1016/j.jns.2021.117355

44. Kang L, Yu H, Yang X, Zhu Y, Bai X, Wang R, et al. Neutrophil Extracellular Traps Released by Neutrophils Impair Revascularization and Vascular Remodeling After Stroke. Nat Commun (2020) 11(1):2488. doi: 10.1038/ s41467-020-16191-y

45. Hoang S, Liauw J, Choi M, Choi M, Guzman RG, Steinberg GK. Netrin-4 Enhances Angiogenesis and Neurologic Outcome After Cerebral Ischemia. J Cereb Blood Flow Metab (2009) 29(2):385-97. doi: 10.1038/jcbfm.2008.128

46. Mostany R, Chowdhury TG, Johnston DG, Portonovo SA, Carmichael ST, Portera-Cailliau C. Local Hemodynamics Dictate Long-Term Dendritic Plasticity in Peri-Infarct Cortex. J Neurosci (2010) 30(42):14116-26. doi: 10.1523/ jneurosci.3908-10.2010

47. Kim SW, Lee H, Lee HK, Kim ID, Lee JK. Neutrophil Extracellular Trap Induced by HMGB1 Exacerbates Damages in the Ischemic Brain. Acta Neuropathol Commun (2019) 7(1):94. doi: 10.1186/s40478-019-0747-x

48. Kim SW, Lee JK. Role of HMGB1 in the Interplay Between NETosis and Thrombosis in Ischemic Stroke: A Review. Cells (2020) 9(8). doi: 10.3390/ cells 9081794

49. Jauch EC, Saver JL, Adams HPJr., Bruno A, Connors JJ, Demaerschalk BM, et al. Guidelines for the Early Management of Patients With Acute Ischemic Stroke: A Guideline for Healthcare Professionals From the American Heart Association/American Stroke Association. Stroke (2013) 44(3):870-947. doi: 10.1161/STR.0b013e318284056a

50. National Institute of Neurological Disorders and Stroke rt-PA Stroke Study Group. Tissue Plasminogen Activator for Acute Ischemic Stroke. N Engl J Med (1995) 333(24):1581-7. doi: 10.1056/nejm199512143332401

51. Tanne D, Kasner SE, Demchuk AM, Koren-Morag N, Hanson S, Grond M, et al. Markers of Increased Risk of Intracerebral Hemorrhage After Intravenous Recombinant Tissue Plasminogen Activator Therapy for Acute Ischemic Stroke in Clinical Practice: The Multicenter Rt-PA Stroke Survey. Circulation (2002) 105(14):1679-85. doi: 10.1161/01.cir. $0000012747.53592 .6 \mathrm{a}$

52. Wang R, Zhu Y, Liu Z, Chang L, Bai X, Kang L, et al. Neutrophil Extracellular Traps Promote tPA-Induced Brain Hemorrhage via cGAS in Mice With Stroke. Blood (2021) 138(1):91-103. doi: 10.1182/blood. 2020008913

53. Chu HX, Kim HA, Lee S, Moore JP, Chan CT, Vinh A, et al. Immune Cell Infiltration in Malignant Middle Cerebral Artery Infarction: Comparison With Transient Cerebral Ischemia. J Cereb Blood Flow Metab (2014) 34 (3):450-9. doi: 10.1038/jcbfm.2013.217

54. Vallés J, Lago A, Santos MT, Latorre AM, Tembl JI, Salom JB, et al. Neutrophil Extracellular Traps are Increased in Patients With Acute Ischemic Stroke: Prognostic Significance. Thromb Haemost (2017) 117 (10):1919-29. doi: 10.1160/th17-02-0130

55. Qureshi AI, Tuhrim S, Broderick JP, Batjer HH, Hondo H, Hanley DF. Spontaneous Intracerebral Hemorrhage. N Engl J Med (2001) 344(19):145060. doi: 10.1056/nejm200105103441907

56. Pinho J, Costa AS, Araújo JM, Amorim JM, Ferreira C. Intracerebral Hemorrhage Outcome: A Comprehensive Update. J Neurol Sci (2019) 398:54-66. doi: 10.1016/j.jns.2019.01.013

57. Puy L, Corseaux D, Perbet R, Deramecourt V, Cordonnier C, Bérézowski V. Neutrophil Extracellular Traps (NETs) Infiltrate Haematoma and Surrounding Brain Tissue After Intracerebral Haemorrhage: A PostMortem Study. Neuropathol Appl Neurobiol (2021):867-77. doi: 10.1111/ nan. 12733

58. Orbán-Kálmándi R, Árokszállási T, Fekete I, Fekete K, Héja M, Tóth J, et al. A Modified In Vitro Clot Lysis Assay Predicts Outcomes in Non-Traumatic Intracerebral Hemorrhage Stroke Patients-The IRONHEART Study. Front Neurol (2021) 12:613441. doi: 10.3389/fneur.2021.613441

59. Zeng H, Fu X, Cai J, Sun C, Yu M, Peng Y, et al. Neutrophil Extracellular Traps may be a Potential Target for Treating Early Brain Injury in 
Subarachnoid Hemorrhage. Transl Stroke Res (2021). doi: 10.1007/s12975021-00909-1

60. Hanhai Z, Bin Q, Shengjun Z, Jingbo L, Yinghan G, Lingxin C, et al. Neutrophil Extracellular Traps, Released From Neutrophil, Promote Microglia Inflammation and Contribute to Poor Outcome in Subarachnoid Hemorrhage. Aging (Albany NY) (2021) 13(9):13108-23. doi: 10.18632/aging.202993

61. Vaibhav K, Braun M, Alverson K, Khodadadi H, Kutiyanawalla A, Ward A, et al. Neutrophil Extracellular Traps Exacerbate Neurological Deficits After Traumatic Brain Injury. Sci Adv (2020) 6(22):eaax8847. doi: 10.1126/sciadv. aax8847

62. Zhu K, Zhu Y, Hou X, Chen W, Qu X, Zhang Y, et al. NETs Lead to Sympathetic Hyperactivity After Traumatic Brain Injury Through the LL37Hippo/MST1 Pathway. Front Neurosci (2021) 15:621477. doi: 10.3389/ fnins.2021.621477

63. Oggioni M, Mercurio D, Minuta D, Fumagalli S, Popiolek-Barczyk K, Sironi M, et al. Long Pentraxin PTX3 Is Upregulated Systemically and Centrally After Experimental Neurotrauma, But Its Depletion Leaves Unaltered Sensorimotor Deficits or Histopathology. Sci Rep (2021) 11(1):9616. doi: $10.1038 / s 41598-021-89032-7$

64. Shindo A, Maki T, Mandeville ET, Liang AC, Egawa N, Itoh K, et al. Astrocyte-Derived Pentraxin 3 Supports Blood-Brain Barrier Integrity Under Acute Phase of Stroke. Stroke (2016) 47(4):1094-100. doi: 10.1161/ strokeaha.115.012133

65. Ravizza T, Moneta D, Bottazzi B, Peri G, Garlanda C, Hirsch E, et al. Dynamic Induction of the Long Pentraxin PTX3 in the CNS After Limbic Seizures: Evidence for a Protective Role in Seizure-Induced Neurodegeneration. Neuroscience (2001) 105(1):43-53. doi: 10.1016/s03064522(01)00177-4

66. Casula M, Montecucco F, Bonaventura A, Liberale L, Vecchié A, Dallegri F, et al. Update on the Role of Pentraxin 3 in Atherosclerosis and Cardiovascular Diseases. Vascul Pharmacol (2017) 99:1-12. doi: 10.1016/ j.vph.2017.10.003

67. Carrizzo A, Procaccini C, Lenzi P, Fusco C, Villa F, Migliarino S, et al. PTX3: An Inflammatory Protein Modulating Ultrastructure and Bioenergetics of Human Endothelial Cells. Immun Ageing (2019) 16:4. doi: 10.1186/s12979019-0144-0

68. Querfurth HW, LaFerla FM. Alzheimer's Disease. N Engl J Med (2010) 362 (4):329-44. doi: 10.1056/NEJMra0909142

69. Heneka MT, Carson MJ, El Khoury J, Landreth GE, Brosseron F, Feinstein DL, et al. Neuroinflammation in Alzheimer's Disease. Lancet Neurol (2015) 14(4):388-405. doi: 10.1016/s1474-4422(15)70016-5

70. Wyss-Coray T, Rogers J. Inflammation in Alzheimer Disease-a Brief Review of the Basic Science and Clinical Literature. Cold Spring Harb Perspect Med (2012) 2(1):a006346. doi: 10.1101/cshperspect.a006346

71. Zlokovic BV. Neurovascular Pathways to Neurodegeneration in Alzheimer's Disease and Other Disorders. Nat Rev Neurosci (2011) 12(12):723-38. doi: $10.1038 / \mathrm{nrn} 3114$

72. Pietronigro EC, Della Bianca V, Zenaro E, Constantin G. NETosis in Alzheimer's Disease. Front Immunol (2017) 8:211. doi: 10.3389/fimmu. 2017.00211

73. Zenaro E, Pietronigro E, Della Bianca V, Piacentino G, Marongiu L, Budui S, et al. Neutrophils Promote Alzheimer's Disease-Like Pathology and Cognitive Decline via LFA-1 Integrin. Nat Med (2015) 21(8):880-6. doi: $10.1038 / \mathrm{nm} .3913$

74. Allen C, Thornton P, Denes A, McColl BW, Pierozynski A, Monestier M, et al. Neutrophil Cerebrovascular Transmigration Triggers Rapid Neurotoxicity Through Release of Proteases Associated With Decondensed DNA. J Immunol (2012) 189(1):381-92. doi: 10.4049/ jimmunol.1200409

75. Grammas P, Samany PG, Thirumangalakudi L. Thrombin and Inflammatory Proteins are Elevated in Alzheimer's Disease Microvessels: Implications for Disease Pathogenesis. J Alzheimers Dis (2006) 9(1):51-8. doi: 10.3233/jad-2006-9105

76. Yin X, Wright J, Wall T, Grammas P. Brain Endothelial Cells Synthesize Neurotoxic Thrombin in Alzheimer's Disease. Am J Pathol (2010) 176 (4):1600-6. doi: 10.2353/ajpath.2010.090406
77. Forlenza OV, Diniz BS, Talib LL, Mendonça VA, Ojopi EB, Gattaz WF, et al. Increased Serum IL-1beta Level in Alzheimer's Disease and Mild Cognitive Impairment. Dement Geriatr Cognit Disord (2009) 28(6):507-12. doi: $10.1159 / 000255051$

78. Alvarez A, Cacabelos R, Sanpedro C, García-Fantini M, Aleixandre M. Serum TNF-Alpha Levels are Increased and Correlate Negatively With Free IGF-I in Alzheimer Disease. Neurobiol Aging (2007) 28(4):533-6. doi: 10.1016/j.neurobiolaging.2006.02.012

79. Zuliani G, Ranzini M, Guerra G, Rossi L, Munari MR, Zurlo A, et al. Plasma Cytokines Profile in Older Subjects With Late Onset Alzheimer's Disease or Vascular Dementia. J Psychiatr Res (2007) 41(8):686-93. doi: 10.1016/ j.jpsychires.2006.02.008

80. Grammas P, Ovase R. Inflammatory Factors Are Elevated in Brain Microvessels in Alzheimer's Disease. Neurobiol Aging (2001) 22(6):83742. doi: 10.1016/s0197-4580(01)00276-7

81. Serebrovska ZO, Serebrovska TV, Kholin VA, Tumanovska LV, Shysh AM, Pashevin DA, et al. Intermittent Hypoxia-Hyperoxia Training Improves Cognitive Function and Decreases Circulating Biomarkers of Alzheimer's Disease in Patients With Mild Cognitive Impairment: A Pilot Study. Int J Mol Sci (2019) 20(21):5405. doi: 10.3390/ijms20215405

82. Vukic V, Callaghan D, Walker D, Lue LF, Liu QY, Couraud PO, et al. Expression of Inflammatory Genes Induced by Beta-Amyloid Peptides in Human Brain Endothelial Cells and in Alzheimer's Brain is Mediated by the JNK-AP1 Signaling Pathway. Neurobiol Dis (2009) 34(1):95-106. doi: 10.1016/j.nbd.2008.12.007

83. Bianca VD, Dusi S, Bianchini E, Dal Prà I, Rossi F. Beta-Amyloid Activates the O-2 Forming NADPH Oxidase in Microglia, Monocytes, and Neutrophils. A Possible Inflammatory Mechanism of Neuronal Damage in Alzheimer's Disease. J Biol Chem (1999) 274(22):15493-9. doi: 10.1074/ jbc.274.22.15493

84. Azevedo EP, Guimarães-Costa AB, Torezani GS, Braga CA, Palhano FL, Kelly JW, et al. Amyloid Fibrils Trigger the Release of Neutrophil Extracellular Traps (NETs), Causing Fibril Fragmentation by NETAssociated Elastase. J Biol Chem (2012) 287(44):37206-18. doi: 10.1074/ jbc.M112.369942

85. Achilli C, Ciana A, Minetti G. Amyloid-Beta (25-35) Peptide Induces the Release of Pro-Matrix Metalloprotease 9 (Pro-MMP-9) From Human Neutrophils. Mol Cell Biochem (2014) 397(1-2):117-23. doi: 10.1007/ s11010-014-2178-0

86. Itoh Y, Nagase H. Preferential Inactivation of Tissue Inhibitor of Metalloproteinases-1 That is Bound to the Precursor of Matrix Metalloproteinase 9 (Progelatinase B) by Human Neutrophil Elastase. J Biol Chem (1995) 270(28):16518-21. doi: 10.1074/jbc.270.28.16518

87. Wang Y, Rosen H, Madtes DK, Shao B, Martin TR, Heinecke JW, et al. Myeloperoxidase Inactivates TIMP-1 by Oxidizing Its N-Terminal Cysteine Residue: An Oxidative Mechanism for Regulating Proteolysis During Inflammation. J Biol Chem (2007) 282(44):31826-34. doi: 10.1074/ jbc.M704894200

88. Kretzschmar GC, Bumiller-Bini V, Gasparetto Filho MA, Zonta YR, Yu KST, de Souza RLR, et al. Neutrophil Extracellular Traps: A Perspective of Neuroinflammation and Complement Activation in Alzheimer's Disease. Front Mol Biosci (2021) 8:630869. doi: 10.3389/fmolb.2021.630869

89. Chiti F, Dobson CM. Protein Misfolding, Functional Amyloid, and Human Disease. Annu Rev Biochem (2006) 75:333-66. doi: 10.1146/annurev. biochem.75.101304.123901

90. Villanueva E, Yalavarthi S, Berthier CC, Hodgin JB, Khandpur R, Lin AM, et al. Netting Neutrophils Induce Endothelial Damage, Infiltrate Tissues, and Expose Immunostimulatory Molecules in Systemic Lupus Erythematosus. J Immunol (2011) 187(1):538-52. doi: 10.4049/jimmunol.1100450

91. Le Joncour A, Martos R, Loyau S, Lelay N, Dossier A, Cazes A, et al. Critical Role of Neutrophil Extracellular Traps (NETs) in Patients With Behcet's Disease. Ann Rheum Dis (2019) 78(9):1274-82. doi: 10.1136/annrheumdis2018-214335

92. Naegele M, Tillack K, Reinhardt S, Schippling S, Martin R, Sospedra M. Neutrophils in Multiple Sclerosis are Characterized by a Primed Phenotype. J Neuroimmunol (2012) 242(1-2):60-71. doi: 10.1016/j.jneuroim. 2011.11.009 
93. Sospedra M, Martin R. Immunology of Multiple Sclerosis. Annu Rev Immunol (2005) 23:683-747. doi: 10.1146/annurev.immunol.23. 021704.115707

94. De Bondt M, Hellings N, Opdenakker G, Struyf S. Neutrophils: Underestimated Players in the Pathogenesis of Multiple Sclerosis (Ms). Int J Mol Sci (2020) 21(12):4558. doi: 10.3390/ijms21124558

95. Tillack K, Naegele M, Haueis C, Schippling S, Wandinger KP, Martin R, et al. Gender Differences in Circulating Levels of Neutrophil Extracellular Traps in Serum of Multiple Sclerosis Patients. J Neuroimmunol (2013) 261(12):108-19. doi: 10.1016/j.jneuroim.2013.05.004

96. Irizar H, Muñoz-Culla M, Sepúlveda L, Sáenz-Cuesta M, Prada Á, CastilloTriviño T, et al. Transcriptomic Profile Reveals Gender-Specific Molecular Mechanisms Driving Multiple Sclerosis Progression. PloS One (2014) 9(2): e90482. doi: 10.1371/journal.pone.0090482

97. Paryzhak S, Dumych T, Mahorivska I, Boichuk M, Bila G, Peshkova S, et al. Neutrophil-Released Enzymes can Influence Composition of Circulating Immune Complexes in Multiple Sclerosis. Autoimmunity (2018) 51(6):297303. doi: 10.1080/08916934.2018.1514390

98. Tay SH, Mak A. Anti-NR2A/B Antibodies and Other Major Molecular Mechanisms in the Pathogenesis of Cognitive Dysfunction in Systemic Lupus Erythematosus. Int J Mol Sci (2015) 16(5):10281-300. doi: 10.3390/ ijms 160510281

99. Takeuchi H, Kawasaki T, Shigematsu K, Kawamura K, Oka N. Neutrophil Extracellular Traps in Neuropathy With Anti-Neutrophil Cytoplasmic Autoantibody-Associated Microscopic Polyangiitis. Clin Rheumatol (2017) 36(4):913-7. doi: 10.1007/s10067-017-3546-4

100. Quail DF, Joyce JA. Microenvironmental Regulation of Tumor Progression and Metastasis. Nat Med (2013) 19(11):1423-37. doi: 10.1038/nm.3394

101. Friebel E, Kapolou K, Unger S, Núñez NG, Utz S, Rushing EJ, et al. SingleCell Mapping of Human Brain Cancer Reveals Tumor-Specific Instruction of Tissue-Invading Leukocytes. Cell (2020) 181(7):1626-1642.e20. doi: 10.1016/ j.cell.2020.04.055

102. Gupta AK, Joshi MB, Philippova M, Erne P, Hasler P, Hahn S, et al. Activated Endothelial Cells Induce Neutrophil Extracellular Traps and are Susceptible to NETosis-Mediated Cell Death. FEBS Lett (2010) 584 (14):3193-7. doi: 10.1016/j.febslet.2010.06.006

103. Huh SJ, Liang S, Sharma A, Dong C, Robertson GP. Transiently Entrapped Circulating Tumor Cells Interact With Neutrophils to Facilitate Lung Metastasis Development. Cancer Res (2010) 70(14):6071-82. doi: 10.1158/ 0008-5472.Can-09-4442

104. McDonald B, Spicer J, Giannais B, Fallavollita L, Brodt P, Ferri LE. Systemic Inflammation Increases Cancer Cell Adhesion to Hepatic Sinusoids by Neutrophil Mediated Mechanisms. Int J Cancer (2009) 125(6):1298-305. doi: 10.1002/ijc.24409

105. Spicer JD, McDonald B, Cools-Lartigue JJ, Chow SC, Giannias B, Kubes P, et al. Neutrophils Promote Liver Metastasis via Mac-1-Mediated Interactions With Circulating Tumor Cells. Cancer Res (2012) 72(16):3919-27. doi: 10.1158/0008-5472.Can-11-2393

106. Tohme S, Yazdani HO, Al-Khafaji AB, Chidi AP, Loughran P, Mowen K, et al. Neutrophil Extracellular Traps Promote the Development and Progression of Liver Metastases After Surgical Stress. Cancer Res (2016) 76 (6):1367-80. doi: 10.1158/0008-5472.Can-15-1591

107. Lin YJ, Wei KC, Chen PY, Lim M, Hwang TL. Roles of Neutrophils in Glioma and Brain Metastases. Front Immunol (2021) 12:701383. doi: 10.3389/fimmu.2021.701383

108. Cools-Lartigue J, Spicer J, McDonald B, Gowing S, Chow S, Giannias B, et al. Neutrophil Extracellular Traps Sequester Circulating Tumor Cells and Promote Metastasis. J Clin Invest (2013) 123(8):3446-58. doi: 10.1172/ jci67484

109. Albrengues J, Shields MA, Ng D, Park CG, Ambrico A, Poindexter ME, et al. Neutrophil Extracellular Traps Produced During Inflammation Awaken Dormant Cancer Cells in Mice. Science (2018) 361(6409):eaao4227. doi: 10.1126/science.aao4227

110. Liu D, Jia S, Sun D, Wang SY, Meng FC, Guo WC. Rapamycin Repairs Damaged Nerve Cells and Neurological Function in Rats With Spinal Cord Injury Through ERK Signaling Pathway. J Biol Regul Homeost Agents (2020) 34(3):865-73. doi: $10.23812 / 20-122-1-45$
111. Zhang X, Guo H, Xie A, Liao O, Ju F. microRNA-331-3p Attenuates Neuropathic Pain Following Spinal Cord Injury via Targeting RAP1A. J Biol Regul Homeost Agents (2020) 34(1):25-37. doi: 10.23812/19-291-a

112. Zha C, Meng X, Li L, Mi S, Qian D, Li Z, et al. Neutrophil Extracellular Traps Mediate the Crosstalk Between Glioma Progression and the Tumor Microenvironment via the HMGB1/RAGE/IL-8 Axis. Cancer Biol Med (2020) 17(1):154-68. doi: 10.20892/j.issn.2095-3941.2019.0353

113. Patel J, Bozeman EN, Selvaraj P. Taming Dendritic Cells With TIM-3: Another Immunosuppressive Strategy Used by Tumors. Immunotherapy (2012) 4(12):1795-8. doi: 10.2217/imt.12.126

114. Tang D, Lotze MT. Tumor Immunity Times Out: TIM-3 and HMGB1. Nat Immunol (2012) 13(9):808-10. doi: 10.1038/ni.2396

115. Chiba S, Baghdadi M, Akiba H, Yoshiyama H, Kinoshita I, Dosaka-Akita H, et al. Tumor-Infiltrating DCs Suppress Nucleic Acid-Mediated Innate Immune Responses Through Interactions Between the Receptor TIM-3 and the Alarmin HMGB1. Nat Immunol (2012) 13(9):832-42. doi: 10.1038/ ni.2376

116. de Mingo Pulido Á, Hänggi K, Celias DP, Gardner A, Li J, Batista-Bittencourt $B$, et al. The Inhibitory Receptor TIM-3 Limits Activation of the cGASSTING Pathway in Intra-Tumoral Dendritic Cells by Suppressing Extracellular DNA Uptake. Immunity (2021) 54(6):1154-67.e7. doi: 10.1016/j.immuni.2021.04.019

117. Curtin JF, Liu N, Candolfi M, Xiong W, Assi H, Yagiz K, et al. HMGB1 Mediates Endogenous TLR2 Activation and Brain Tumor Regression. PloS Med (2009) 6(1):e10. doi: 10.1371/journal.pmed.1000010

118. Schulz C, Gabriel G, von Köckritz-Blickwede M. Detrimental Role of Neutrophil Extracellular Traps During Dengue Virus Infection. Trends Immunol (2020) 41(1):3-6. doi: 10.1016/j.it.2019.11.010

119. Wang Y, Du F, Hawez A, Mörgelin M, Thorlacius H. Neutrophil Extracellular Trap-Microparticle Complexes Trigger Neutrophil Recruitment via High-Mobility Group Protein 1 (HMGB1)-Toll-Like Receptors(TLR2)/TLR4 Signalling. Br J Pharmacol (2019) 176(17):335063. doi: $10.1111 /$ bph. 14765

120. Wynn TA, Chawla A, Pollard JW. Macrophage Biology in Development, Homeostasis and Disease. Nature (2013) 496(7446):445-55. doi: 10.1038/ nature 12034

121. Wong KW, Jacobs WRJr. Mycobacterium Tuberculosis Exploits Human Interferon $\gamma$ to Stimulate Macrophage Extracellular Trap Formation and Necrosis. J Infect Dis (2013) 208(1):109-19. doi: 10.1093/infdis/jit097

122. Daniel C, Leppkes M, Muñoz LE, Schley G, Schett G, Herrmann M. Extracellular DNA Traps in Inflammation, Injury and Healing. Nat Rev Nephrol (2019) 15(9):559-75. doi: 10.1038/s41581-019-0163-2

123. Doster RS, Rogers LM, Gaddy JA, Aronoff DM. Macrophage Extracellular Traps: A Scoping Review. J Innate Immun (2018) 10(1):3-13. doi: 10.1159/ 000480373

124. Liu P, Wu X, Liao C, Liu X, Du J, Shi H, et al. Escherichia Coli and Candida Albicans Induced Macrophage Extracellular Trap-Like Structures With Limited Microbicidal Activity. PloS One (2014) 9(2):e90042. doi: 10.1371/ journal.pone.0090042

125. Je S, Quan H, Yoon Y, Na Y, Kim BJ, Seok SH. Mycobacterium Massiliense Induces Macrophage Extracellular Traps With Facilitating Bacterial Growth. PloS One (2016) 11(5):e0155685. doi: 10.1371/journal.pone.0155685

126. Pertiwi KR, de Boer OJ, Mackaaij C, Pabittei DR, de Winter RJ, Li X, et al. Extracellular Traps Derived From Macrophages, Mast Cells, Eosinophils and Neutrophils are Generated in a Time-Dependent Manner During Atherothrombosis. J Pathol (2019) 247(4):505-12. doi: 10.1002/ path.5212

127. Ziv Y, Ron N, Butovsky O, Landa G, Sudai E, Greenberg N, et al. Immune Cells Contribute to the Maintenance of Neurogenesis and Spatial Learning Abilities in Adulthood. Nat Neurosci (2006) 9(2):268-75. doi: 10.1038/ nn 1629

128. Kettenmann H, Hanisch UK, Noda M, Verkhratsky A. Physiology of Microglia. Physiol Rev (2011) 91(2):461-553. doi: 10.1152/physrev. 00011.2010

129. Wang C, Wang Y, Shi X, Tang X, Cheng W, Wang X, et al. The TRAPs From Microglial Vesicles Protect Against Listeria Infection in the CNS. Front Cell Neurosci (2019) 13:199. doi: 10.3389/fncel.2019.00199 
130. Agrawal I, Sharma N, Saxena S, Arvind S, Chakraborty D, Chakraborty DB, et al. Dopamine Induces Functional Extracellular Traps in Microglia. iScience (2021) 24(1):101968. doi: 10.1016/j.isci.2020.101968

Conflict of Interest: The authors declare that the research was conducted in the absence of any commercial or financial relationships that could be construed as a potential conflict of interest.

Publisher's Note: All claims expressed in this article are solely those of the authors and do not necessarily represent those of their affiliated organizations, or those of the publisher, the editors and the reviewers. Any product that may be evaluated in this article, or claim that may be made by its manufacturer, is not guaranteed or endorsed by the publisher.

Copyright $\odot 2021 \mathrm{Wu}$, Zeng, Cai and Chen. This is an open-access article distributed under the terms of the Creative Commons Attribution License (CC BY). The use, distribution or reproduction in other forums is permitted, provided the original author(s) and the copyright owner(s) are credited and that the original publication in this journal is cited, in accordance with accepted academic practice. No use, distribution or reproduction is permitted which does not comply with these terms. 